\title{
Correction to: Detection of metastases in newly diagnosed prostate cancer by using 68Ga-PSMA PET/CT and its relationship with modified D'Amico risk classification
}

\author{
Duygu Has Simsek ${ }^{1} \cdot$ Yasemin Sanli $^{1} \cdot$ Muge Nur Engin $^{1} \cdot$ Selcuk Erdem $^{2} \cdot$ Oner Sanli $^{2}$
}

Published online: 7 April 2021

(C) Springer-Verlag GmbH Germany, part of Springer Nature 2021

Correction to: Eur J Nucl Med Mol Imaging https://doi.org/10.1007/s00259-020-04995-5

The figure legends are correctly placed, however, figure images are ordered incorrectly during the publishing process.

Fig. $1 \rightarrow$ should be replaced as Fig. 4

Fig. $2 \rightarrow$ should be replaced as Fig. 5

Fig. $3 \rightarrow$ should be replaced as Fig. 6

Fig. $4 \rightarrow$ should be replaced as Fig. 1

Fig. $5 \rightarrow$ should be replaced as Fig. 3

Fig. $6 \rightarrow$ should be replaced as Fig. 2

The correct order of figures are shown on the next page.

The original article has been corrected.

This article is part of the Topical Collection on Erratum

The online version of the original article can be found at https://doi.org/ 10.1007/s00259-020-04995-5

Duygu Has Simsek

dr.duyguhas@hotmail.com

1 Istanbul Faculty of Medicine, Department of Nuclear Medicine, Istanbul University, 34093 İstanbul, Fatih, Turkey

2 Istanbul Faculty of Medicine, Department of Urology, Istanbul University, 34093 İstanbul, Fatih, Turkey 
a

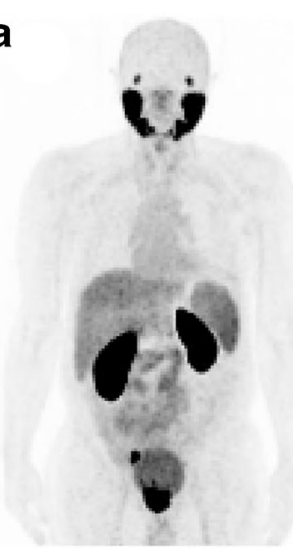

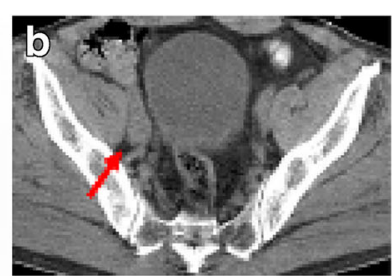

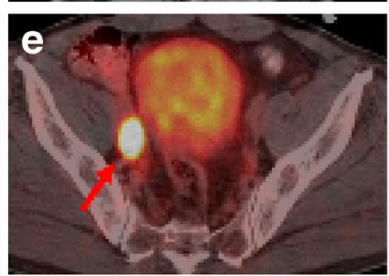

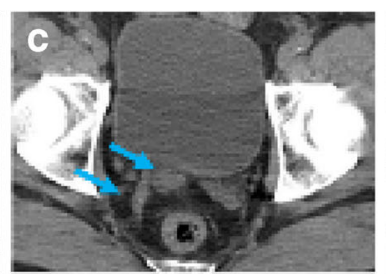

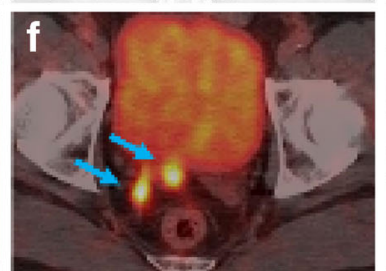

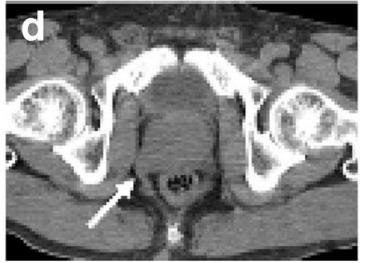

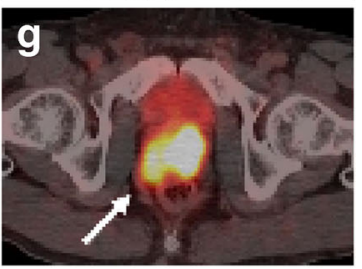

Fig. 1

Fig. 2
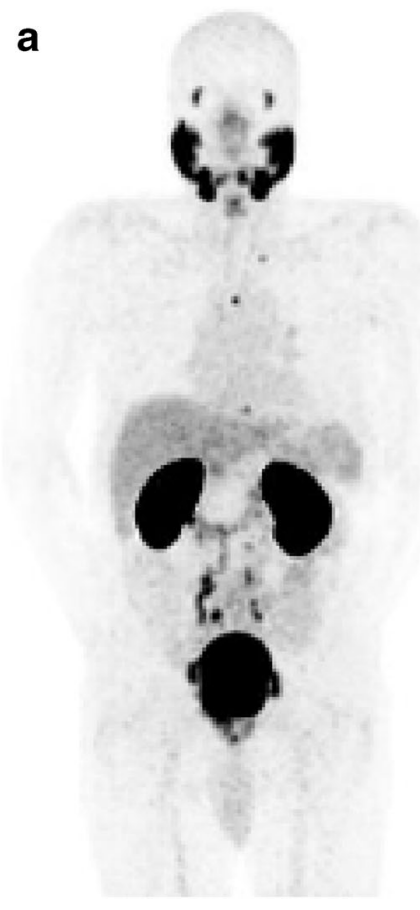
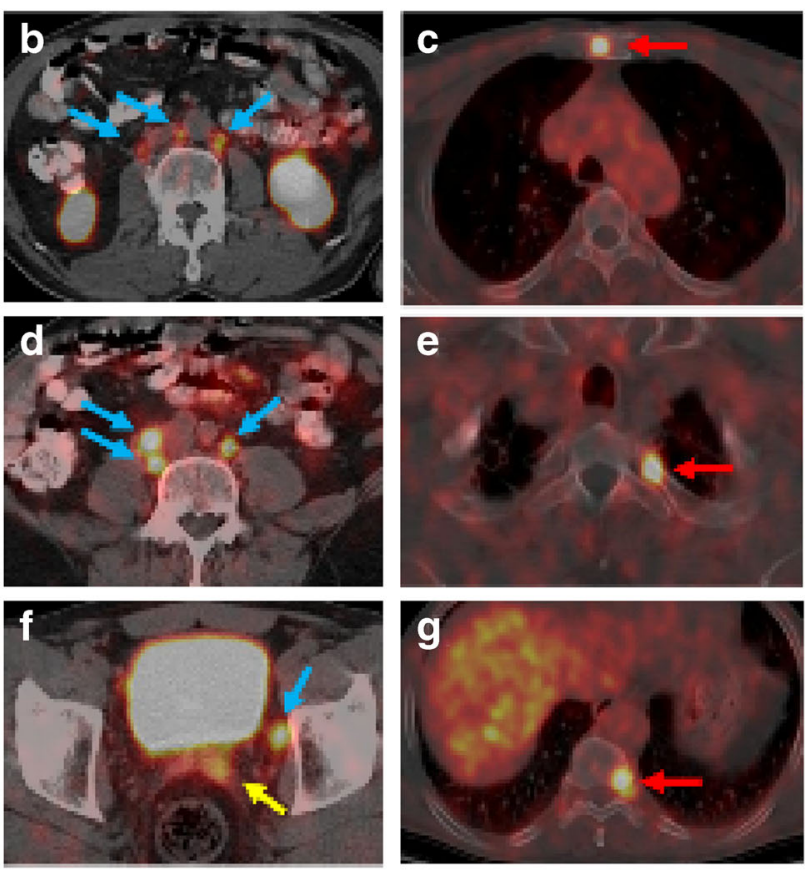
Fig. 3

Fig. 4

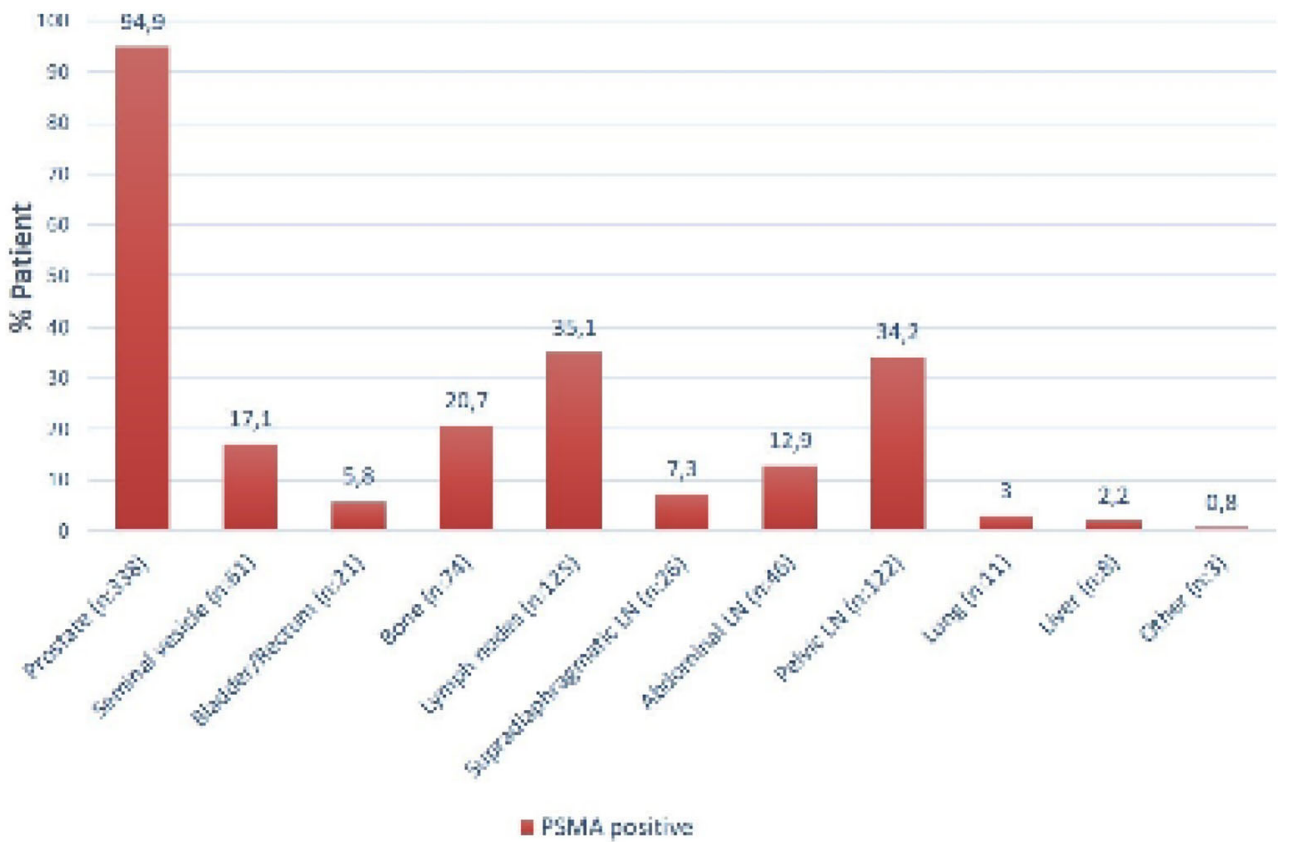



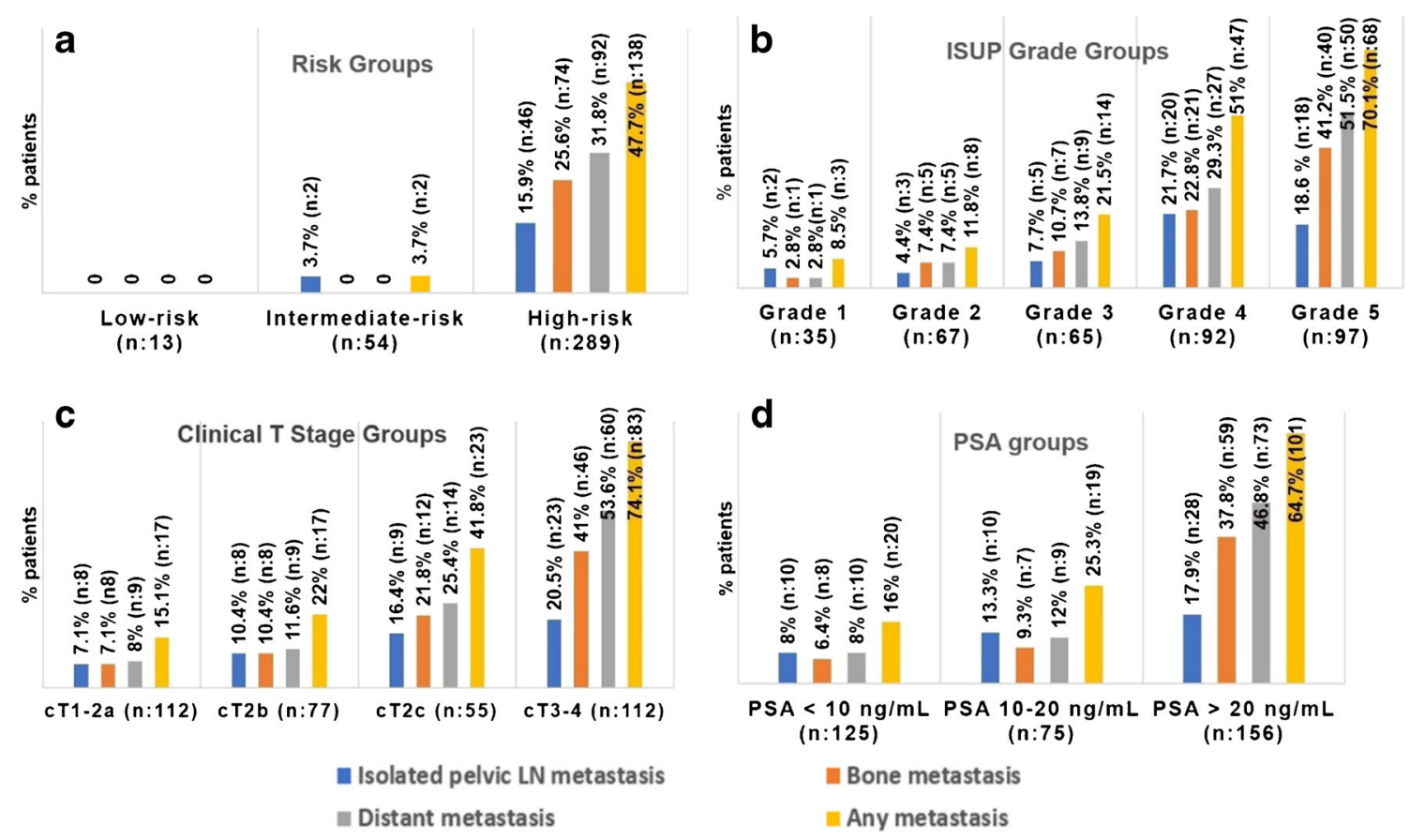

Fig. 5 
Fig. 6 a

Low-risk (n:13)
Intermediate-risk (n:27)
High-risk (n:85)

Patients with PSA $<10 \mathrm{ng} / \mathrm{ml}$

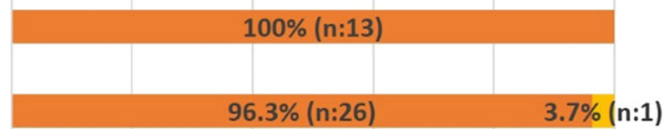

$77.6 \%(n: 66)$

40

60

80

100

120

b

Intermediate-risk (n:27)

High-risk (n:48)

$62.5 \%(n: 30)$

$18.8 \%(n: 9) \quad 18.8 \%(n: 9)$

0

20

40

60

80

100

120

Non-metastatic

- Isolated pelvic nodal metastasis

n Distant metastasis

C

High-risk (n:156)

Patients with PSA $>\mathbf{2 0}$ ng/ml

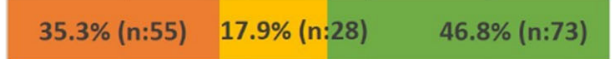

0

20

40

60

80

100

120

- Non-metastatic Isolated pelvic nodal metastasis $\quad$ Distant metastasis

Publisher's note Springer Nature remains neutral with regard to jurisdictional claims in published maps and institutional affiliations. 